\title{
Mandeln raus bei Schlafapnoe im Kindesalter?
}

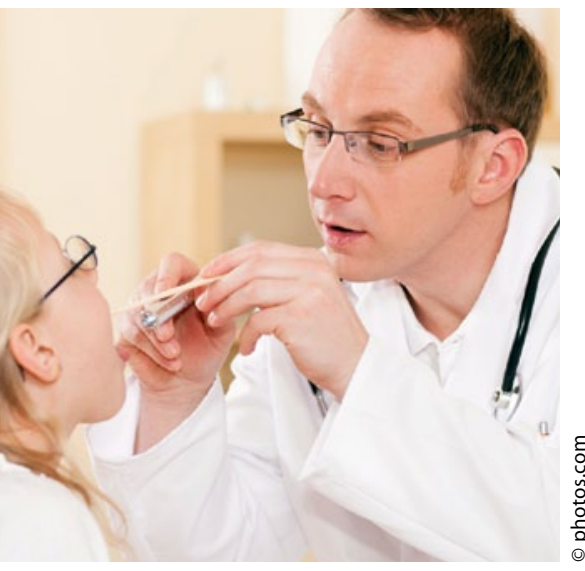

Operieren oder nicht?

Die Entfernung der „Polypen“ führt im Vergleich zum abwartenden Beobachten zwar nicht zu einer testpsychologischen Verbesserung von Aufmerksamkeit und Handlungsfähigkeit, geht aber mit einer Besserung von Verhaltensproblemen und Lebensqualität sowie der polysomnografischen Befunde einher.
- 464 Kinder im Alter zwischen fünf und neun Jahren mit nachgewiesenem obstruktiven Schlaf-Apnoe-Syndrom wurden in eine Gruppe mit früher Adenotonsillektomie und abwartendem Beobachten randomisiert. Vor der Therapie und nach sieben Monaten wurden Polysomnografien sowie neuropsychologische Untersuchungen durchgeführt und die Lebensqualität evaluiert.

Die Scores der neuropsychologischen Untersuchungen änderten sich in beiden Gruppen nicht signifikant. Durch die frühe Adenotonsillektomie wurden aber das Verhalten der Kinder, die Lebensqualität und die polysomnografischen Befunde signifikant günstiger beeinflusst als in der Beobachtungsgruppe. Bei 79\% der operierten Kinder kam es zu einer vollständigen Normalisierung der Polysomnografie. Dies traf bei den nur beobachteten Kindern nur in 46\% der Fälle zu.

- C. L. Marcus et al.

(Korres.: sredline@partners.org): A randomized trial of adenotonsillectomy for childhood sleep apnea. New Engl J Med 2013;368:2366-2376

\section{MMW Kommentar}

Die sorgfältig durchgeführte Studie zeitigte kein Ergebnis, das nur ein Vorgehen zulässt. Die größte Sorge der Eltern, dass die geistige Entwicklung der Kinder durch bloßes abwartendes Beobachten behindert werden könnte, wird durch die Untersuchung nicht bestätigt. Allerdings steigt zumindest im statistischen Mittel durch die Operation die Lebensqualität, und evtl. vorhandene Verhaltensauffälligkeiten der Kinder bessern sich. Die polysomnografischen Befunde werden durch die Operation in höherem Ausmaß verbessert, allerdings kommt es im Verlauf von vier Jahren auch bei fast der Hälfte der nicht operierten Kinder zu einer deutlichen Verbesserung. Die Entscheidung für oder gegen die frühzeitige Operation ist nach wie vor nicht leicht zu treffen, doch hat die Studie zumindest einige wertvolle Anhaltspunkte geliefert, um die Wahl auf solidere Beine zu stellen. Wahrscheinlich spielt aber das „Bauchgefühl" der Eltern und die Einstellung zur Medizin an sich nach wie vor die wichtigste Rolle.

H. S. FüeßI .

\section{Gehen Depressionen auf die Knochen?}

\begin{abstract}
Viele Studien zeigten einen Zusammenhang zwischen klinisch relevanter Depression (Major Depressive Disorder, MDD) und Reduktion der Knochendichte mit der Folge eines erhöhten Osteoporoserisikos. Zum Pathomechanismus kann gegenwärtig nur spekuliert werden.
\end{abstract}

— Bei MDD-Patienten im jungen und mittleren Erwachsenenalter, die im Rahmen einer multizentrischen Studie rekrutiert wurden, wurde die Knochendichte im Bereich der Lendenwirbelsäule und des Schenkelhalses gemessen. $\mathrm{Ne}$ ben dem Depressionsschweregrad (Montgomery Åsberg Depression Rating Scale, MADRS) wurde eine Vielzahl biochemischer, endokrinologischer und immunologischer Marker mit Relevanz für den Knochenstoffwechsel bestimmt.

Nach Ausschluss von 104 Patienten aufgrund von Komorbiditäten wurden 50 Patienten analysiert. Der durchschnittliche Depressionsschweregrad war hoch (MADRS-Score 27,5), und auch die Erkrankungsdauer war im Schnitt eher lang (4,9 Jahre). Mit einer Ausnahme zeigte sich bei keinem Teilnehmer eine relevant erniedrigte Knochendichte.

Somatisch gesunde, akut depressive Patienten im jüngeren und mittleren Lebensalter zeigen keine Osteopenie oder erhöhte osteoklastische Aktivität. Auch die SSRI-Einnahme führt in dieser $\mathrm{Pa}$ tientengruppe nicht zu einer Knochendichteminderung.

\footnotetext{
- Malik Pet al.

Bone mineral density and bone metabolism in patients with major depressive disorder without somatic comorbidities. Prog Neuropsychopharmacol Biol Psychiatry 2013; 44: 58-63
}

\section{MMW Kommentar}

Die vorliegende Studie ist vergleichsweise klein und verfügt über keine Kontrollgruppe, jedoch ist ihr Verdienst die weitgehende Bereinigung von konfundierenden Faktoren durch strenge Ausschlusskriterien. Bei ausreichendem Symptomschweregrad und auch genügend langer Krankheitsdauer erbrachte sie unter diesen Voraussetzungen ein negatives Ergebnis. Als zweites Hauptergebnis ging auch die SSRI-Behandlung nicht mit einer verminderten Knochendichte einher. Diesbezügliche Daten sind bisher nicht eindeutig trotz eines Überwiegens positiver Studien. Zusammengefasst dürfte bei depressiven $\mathrm{Pa}$ tienten das kumulative Risiko mehrerer Faktoren ausschlaggebend für eine Osteopenie sein. Die MDD oder eine SSRI-Behandlung sind möglicherweise keine unabhängigen Risikofaktoren. C. Lange-Asschenfeldt . 